\title{
Los Métodos de Resolución de Problemas y el Desarrollo del Pensamiento Matemático
}

\section{Problem- Solving Methods and Mathematical Thought Development}

\author{
Jorge Antonio Díaz Lozada* \\ Rafael Díaz Fuentes **
}

\begin{abstract}
Resumen
En los últimos años se ha alcanzado cierto consenso acerca del papel de la enseñanza de la Matemática en el desarrollo del pensamiento, por encima de la transferencia de conocimientos matemáticos. En este sentido, la atención al desarrollo de la capacidad para resolver problemas va cediendo terreno con respecto al desarrollo del pensamiento en la resolución de problemas. Numerosos autores han aportado métodos para resolver problemas, sin embargo, aún son escasas las propuestas concretas que ayuden a los docentes a utilizar los métodos de resolución de problemas y los recursos de la heurística para llevar a la práctica el tratamiento de la resolución de problemas con el fin de estimular el desarrollo del pensamiento matemático. Este trabajo analiza las potencialidades de los métodos de resolución de problemas para estimular el desarrollo del pensamiento matemático y propone ideas para su implementación en el aula.
\end{abstract}

Palabras clave: Desarrollo del pensamiento. Heurística. Pensamiento matemático. Métodos de resolución de problemas.

\begin{abstract}
In recent years, we have reached a certain consensus about the role of teaching mathematics in the development of thought, above the transfer of mathematical knowledge. In this sense, the attention to the development of the ability to solve problems is giving way to thought development in problem-solving. Many authors have provided methods to solve problems; however, the are still limited concrete proposals to help teachers use methods of problem-solving and heuristics resources to implement the treatment of solving problems in order to stimulate the development of mathematical thinking. This paper analyzes the potential of problem-solving methods to stimulate the development of mathematical thinking and proposes ideas for its implementation in the classroom.
\end{abstract}

Keywords: Though development. Heuristic. Mathematical thought. Problemsolving methods.

\section{Introducción}

La resolución de problemas es una situación de aprendizaje bien conocida y polémica, lo mismo para estudiantes, padres y docentes; en el caso de los primeros, por sus

\footnotetext{
* Doctor en Ciencias Pedagógicas por la Universidad Pedagógica de La Habana, Profesor Auxiliar en la Universidad Tecnológica de La Habana, Cuba. Dirección Postal: Avenida 114 y autopista de Pinar del Rio, Marianao, La Habana. E-mail: jorgedlz@ yandex.com.

${ }^{* *}$ Rafael Díaz Fuentes, Master en Ciencias Matemáticas, Investigador en el Instituto de Cibernética, Matemática y Física. Dirección Postal: Calle E esquina 15, Vedado, La Habana, Cuba. Aspirante a Doctor en la Universidad de Insubria, Como, Italia. E-mail: rafaeldfu@gmail.com.
} 
dificultades para resolverlos al parecer insuperables, con respecto a los segundos, por los ratos buenos o malos que, en otros tiempos, les hicieron pasar en la escuela y las referencias de las dificultades de sus hijos, y, para los terceros, por ser motivo de fracaso escolar en el proceso de enseñanza aprendizaje, sobre todo de la Matemática.

Varios investigadores han identificado al importante papel de la resolución de problemas en el proceso de enseñanza aprendizaje. Sin embargo, las potencialidades de esta situación de aprendizaje no son aprovechadas lo suficiente, se observa un marcado énfasis en que los alumnos se apropien de patrones que los lleven a desarrollar la capacidad para resolver problemas y no se tiene en cuenta su papel en el desarrollo del pensamiento y, sobre todo, del pensamiento matemático.

Pero, ¿cómo organizar la enseñanza de la Matemática y en especial la resolución de problemas de modo que más que ofrecer patrones para resolver problemas aproveche sus potencialidades para estimular el desarrollo del pensamiento matemático?

\section{La resolución de problemas y el pensamiento matemático}

En los últimos años, diferentes investigadores, han coincidido en que el trabajo con los problemas matemáticos en la escuela merece ocupar un papel central en el proceso de enseñanza, tanto en la Matemática como en otras asignaturas. A partir de las investigaciones más cercanas en el tiempo se identifican carencias que presentan los estudiantes en el proceso de resolución de problemas:

$\checkmark$ Dificultades en la comprensión de los problemas que no permiten una adecuada búsqueda de la vía de solución (CAPOTE, 2003).

$\checkmark$ Incoherencias en las respuestas a los problemas y bloqueos en el proceso de búsqueda de la vía de solución (VILA-CORTS, 2001).

$\checkmark$ Inhibición en la búsqueda de la vía de solución a ciertos problemas como resultado del efecto negativo de experiencias anteriores (GUILERA, 2002).

$\checkmark$ Escasa autorregulación de los procesos mentales por los estudiantes en la resolución de problemas (ZUFFI; ONUCHIC, 2007).

Por otra parte, desde la posición de los docentes entre las dificultades más notables se observan:

El poco tiempo que se brinda a los estudiantes para resolver los problemas, lo que no estimula la reflexión (GUILERA, 2002). 
$\checkmark$ Marcado énfasis en la función que desempeñan los problemas matemáticos como medio de asimilación o fijación de conocimientos, sin aprovechar las potencialidades que brindan al desarrollo del pensamiento (SUÁREZ, 2003).

Estas carencias o dificultades son consecuencia del escaso aprovechamiento de las potencialidades de la resolución de problemas para favorecer la actividad mental de los estudiantes, y justifican la necesidad de indagar acerca de su tratamiento metodológico con un enfoque desarrollador, que brinde a los docentes propuestas concretas para mejorarlas.

A través de los años, la mayor preocupación de los docentes e investigadores ha sido encontrar la vía o método para llegar a la solución del problema, a partir del método de Polya (1973) aparecen diversas propuestas inspiradas en esta (MÜLLER, 1978; JUNGK, 1982; SCHOENFELD, 1985; KRULIK; RUDNICK, 1988; SANTOS, 1993).

Müller (1978) y Jungk (1982) conciben todo un sistema teórico que denominan instrucción heurística, que incluye procedimientos para facilitar la búsqueda de la vía de solución y que se integran en un programa o sistema de procedimientos que incluye:

\section{Orientación hacia el problema,}

2. trabajo en el problema,

3. solución del problema,

4. evaluación de la solución y la vía.

Otra propuesta es la de Schoenfeld (1985), que centra la atención en la relación entre la resolución de problemas y el desarrollo del pensamiento, y propone un método para el proceso de resolución:

\section{Comprensión del problema,}

2. diseño de un plan de solución,

3. ejecutar el plan,

4. mirada retrospectiva.

Krulik y Rudnick (1988) proponen un modelo dirigido a superar las insuficiencias en la enseñanza de la matemática, conciben la resolución de problemas como una habilidad y plantean al desarrollo del pensamiento como lo más importante en el proceso de resolución, el que estructuran en etapas:

1. Lectura del problema,

2. exploración,

3. selección de una estrategia,

4. resolver el problema,

5. vista retrospectiva y extrapolación a otros problemas. 
Santos (1993) modela el proceso de resolución en ocho fases, que se inspiran en el modo en que se desarrolla una investigación científica:

1. Consciencia de la existencia del problema,

2. supresión de los dados,

3. interés por la situación problemática abordada,

4. análisis cualitativo,

5. formulación de hipótesis,

6. estrategias de resolución,

7. análisis de los resultados,

8. maduración.

Un análisis de estas propuestas permite identificar como tendencias: su papel en el desarrollo de la capacidad para resolver problemas y para el desarrollo del pensamiento como esencia de esta actividad.

El dinamismo, rapidez y volumen con que se generan conocimientos en la actualidad plantean nuevos retos a los sistemas educativos. Los procesos de pensamiento, por ser más estables que los contenidos declarativos, deben ser considerados como lo más importante que se le trasmite a las nuevas generaciones.

La enseñanza de la Matemática donde predomina el método sobre el resto de los contenidos constituye un excelente espacio para lograr los fines señalados. Sausen y Guérios (2010) señalan que una de las metas de la enseñanza de la Matemática es estimular a los estudiantes a pensar de manera fecunda, propiciar el razonamiento lógico, de modo eficaz e inteligente, que luego le permita resolver situaciones diversas tanto en la escuela como fuera de esta.

Esta disciplina, además de su valor como herramienta empleada en otras ciencias, constituye un modelo de pensamiento científico sustentado en principios sólidos. Posee características que le son propias: empleo de expresiones lógicas y concretas, la necesidad de reflexión, el uso de una simbología precisa y coherente y el manejo de procesos que influyen, de manera significativa, en el modo de pensar de los estudiantes.

Es por esto que se considera portadora de una forma de pensar característica, que ha sido objeto de análisis por parte de diferentes autores ocupados en su enseñanza que se conoce como pensamiento matemático.

Según el NCTM (2004) el pensamiento matemático es un elemento importante en la preparación de los profesionales, técnicos u hombres y mujeres en sentido general. Este reconocimiento es consecuencia del impetuoso desarrollo de las nuevas tecnologías de la 
información y las comunicaciones, y razón por la que tanto la sociedad como los sistemas educativos deben precisar hasta qué nivel debe desarrollarse, atendiendo a las necesidades de la sociedad.

En opinión de Koliaguin (1975), son rasgos del pensamiento matemático: la profundidad, la amplitud, el carácter autocrítico del pensamiento y la flexibilidad. Esta caracterización intenta resumir el modo matemático de pensar, centrándose en capacidades necesarias para la actividad matemática sin reparar en el conocimiento con que se opera.

El Ministerio de Educación de la República de Cuba (1980) además de reconocer el papel del pensamiento matemático en la formación integral de los estudiantes, identifica como rasgos fundamentales: la movilidad, rapidez, la posibilidad de cambiar de una operación mental a otra, de abarcar estructuras formales, la racionalización del proceso de reflexión mental para llegar al resultado, entre otras. Esta caracterización abarca capacidades matemáticas, destacando los aspectos lógico-deductivos y, en menor medida, heurísticos.

Jungk (1982) identifica, entre otros rasgos del pensamiento matemático: el lógicodeductivo, el pensamiento creativo y con fantasía, la formación lingüística y el pensamiento final; aquí, es importante destacar el pensamiento con fantasía necesario para la estimación, para prever lo que es posible y lo que no lo es y que es propio de los procesos creativos en que se desempeña la actividad del profesional de las ciencias técnicas.

Para Schoenfeld (1992) pensar matemáticamente es: investigar soluciones, no memorizar procedimientos; explorar patrones, no memorizar fórmulas, formular conjeturas, no hacer ejercicios. Considera que el pensamiento matemático se puede caracterizar con cuatro rasgos: el dominio del conocimiento o recursos, los métodos heurísticos, el control y el sistema de creencias.

En esta caracterización se incluyen aspectos relacionados con la heurística y la lógica, pero considera además aspectos del orden subjetivo como las creencias y los criterios personales, necesarios para resolver problemas.

La caracterización del pensamiento matemático propuesta por Ballester y otros (2001) tiene un espectro amplio, que agrega a los rasgos anteriores: el pensamiento geométrico espacial, el algorítmico, el pensamiento funcional y la racionalización del trabajo mental. Esta caracterización lo aborda en un espectro amplio de capacidades asociadas a la disciplina y destaca a la resolución de problemas como contexto de aplicación, e incluye algunos contenidos característicos del conocimiento matemático que condicionan la forma matemática de pensar. 
De acuerdo con Fernández (2003), es propio del pensamiento matemático: la exploración de pluralidad de alternativas con coherencia lógica, la búsqueda de relaciones y el empleo de acciones mentales adecuadas para cada situación. Esta caracterización contempla los procesos lógicos, los heurísticos y la actividad metacognitiva, tres esferas esenciales en la resolución de problemas.

Otra caracterización del pensamiento matemático es propuesta por Rodríguez (2003), que lo considera como una capacidad que permite interpretar información en la vida diaria, tomar decisiones en función de esa interpretación, el uso de las herramientas matemáticas incluyendo la modelación, un pensamiento analítico, crítico y flexible, tanto al razonar como al valorar razonamientos de otros.

Onuchic y Allevato (2004) relacionan el pensamiento matemático con el establecimiento de relaciones entre conocimientos, saber comunicar estas relaciones, desarrollar razonamientos, la capacidad de resolver problemas y de proponer otros. Así, hacen referencia a aspectos como: el razonamiento, la búsqueda de relaciones, el empleo del formalismo matemático, la resolución e identificación de problemas.

A partir de estas caracterizaciones de pensamiento matemático se identifican tres dimensiones esenciales:

- el razonamiento lógico-deductivo,

- la heurística como recurso de búsqueda,

- la metacognición que permite valorar la actividad mental que se realiza.

La medición de estas dimensiones esenciales del pensamiento matemático necesita que se asuman indicadores que permitan su evaluación de manera tangible. En cuanto al razonamiento lógico-deductivo:

- Aplicar conceptos y proposiciones,

- organizar y representar la información que brinda el problema,

- deducir consecuencias de los datos del problema,

- $\quad$ argumentar y demostrar proposiciones.

Con respecto a la heurística se asumen como indicadores:

- Identificar nexos y relaciones,

- variar las condiciones iniciales del problema,

- identificar casos especiales y casos límites,

- explorar diferentes vías de solución.

En cuanto a la metacognición se toman como indicadores:

- Evaluar los pasos que se realizan, 
- controlar la ejecución de la vía de solución,

- reflexionar acerca de la vía de solución,

- identificar alternativas de vías de solución y,

- lograr precisión en la estructuración de la vía de solución.

En opinión de Jungk (1982), el desarrollo del pensamiento matemático transcurre en niveles que se corresponden con el desarrollo de la Matemática como ciencia:

1. Operaciones con objetos concretos, como: conjuntos y figuras geométricas, obtención de propiedades por inducción, la identificación a partir del reconocimiento de propiedades,

2. ordenamiento lógico, atendiendo a propiedades,

3. deducciones que preparan al estudiante para asimilar teorías axiomáticas,

4. aprendizaje de sistemas deductivos abstractos.

El pensamiento matemático permite reflejar el mundo objetivo por medio de los conceptos, relaciones, procedimientos de cuantificación y modelación abstraídos de la realidad y, en especial, buscar solución a los problemas.

Es evidente la relación entre la resolución de problemas y el desarrollo del pensamiento matemático. Según el NCTM (2010), en la resolución de problemas se incita al estudiante a reflejar su pensamiento de modo que puedan aplicar y adaptar estrategias que puedan transferir a otros problemas y en otros contextos, desarrollando la perseverancia y curiosidad por la actividad resolutora.

Al respecto, es obvio que el estudiante despliega y desarrolla el pensamiento cuando resuelve problemas, pero, a su vez, está en mejores condiciones para resolver problemas cuando alcanza un adecuado nivel de desarrollo en el pensamiento matemático.

\section{La heurística y la resolución de problemas}

La enseñanza consciente, planificada y científica de reglas, procedimientos y principios para la exploración y búsqueda de solución a tareas docentes o problemas ha sido denominada por algunos autores instrucción heurística (JUNGK, 1982; MÜLLER, 1978; BALLESTER et al., 2001), se sustenta en la aplicación de programas heurísticos, auxiliado con medios, reglas, y procedimientos.

Los elementos que conforman la heurística son conocidos desde la antigüedad, sin embargo en la resolución de problemas aún no se aprovechan lo suficiente todas sus potencialidades (JUNGK, 1982; RON, 2007). La heurística facilita al docente conducir al 
estudiante al descubrimiento de suposiciones, hipótesis y reglas, de forma independiente, a través de impulsos que movilicen su actividad mental.

Entre los procedimientos propios de la heurística se encuentran los principios heurísticos generales: el de analogía, el de reducción y el de inducción (MÜLLER, 1978; BALLESTER et al., 2001). Es importante destacar el de analogía, muy útil para estimular a los estudiantes para que descubran proposiciones, sugerirles el empleo de determinados métodos, procedimientos, o la vía de solución de un problema, a partir de la comparación de las semejanzas entre las estructuras interna y externa de los problemas.

El de reducción posibilita la transformación de un problema desconocido a partir de otro ya conocido, la elaboración de un modelo que represente el problema de forma más conveniente, la búsqueda de proposiciones generales a partir de resultados particulares.

Existen, además, principios especiales, entre otros: el principio de generalización, el principio de movilidad, el principio de medir y probar sistemáticamente y el principio de consideración de casos especiales y casos límites.

Polya (1973) reconoce el papel de las preguntas que puede formular el docente en forma de reglas o procedimientos para impulsar la actividad mental en la búsqueda de la vía de solución, estas contienen acciones y operaciones a realizar por el estudiante, pueden darse como indicaciones, sugerencias o simplemente como preguntas que movilizan la actividad mental. Sobre la base de esta concepción Jungk (1982) valora estas preguntas, que identifica como impulsos heurísticos con un importante papel para estimular la actividad mental y el pensamiento de los alumnos.

Estos impulsos constituyen la herramienta que puede utilizar el docente para estimular la actividad mental del estudiante y lograr que, primero en el plano externo transiten por cada una de las dimensiones del pensamiento matemático para que, luego, internalicen las habilidades propias de cada dimensión y las incorporen a su actividad mental en un nivel superior de desarrollo del pensamiento matemático.

Otros procedimientos heurísticos son las estrategias de búsqueda, que constituyen el método principal para identificar los medios matemáticos que se necesitan para la idea fundamental de solución del problema (MÜLLER, 1978; JUNGK, 1982; BALLESTER et al., 2001). Existen dos que pueden ser aplicadas a cualquier tipo de ejercicio o problema, la primera: el trabajo hacia adelante, que consiste en partir de los datos y a través de inferencias y deducciones llegar a la solución.

La segunda, de trabajo hacia atrás se realiza con el análisis del problema a partir de lo que se busca, para identificar relaciones entre las exigencias del problema y la información de 
que se dispone, de modo que se identifiquen objetivos parciales o los resultados intermedios que habría que plantearse para encontrar la vía de solución. Al respecto, es importante destacar que cuando se es capaz de invertir el proceso de inferencias desde la exigencia hasta los datos se está en mejores condiciones de rastrear los nexos que vinculan la información inicial con el resultado al que se aspira a llegar, desarrollando una actividad mental intensa.

En realidad, ambas estrategias son importantes en la resolución de problemas porque sirven como un esquema general de partida para organizar la búsqueda de vías de solución, el resolutor debe valorar cuando trabajar con una o con la otra.

Según Wang (2012) el pensamiento matemático se desarrolla a través de la formación y desarrollo de hábitos que son necesarios en la resolución de problemas. En la opinión de los autores de la presente investigación, el mejor recurso para estimular el desarrollo del pensamiento son los métodos de resolución de problemas, siempre que tengan en cuenta todas las dimensiones del pensamiento matemático a través de cada uno de los momentos de la actividad resolutora.

El empleo de procedimientos heurísticos se organiza en métodos como los ya referidos, que permiten organizar el proceso de búsqueda de la vía de solución. Estos se estructuran, generalmente, en cuatro fases que incluyen: la comprensión, la elaboración de un plan, la ejecución del plan y la evaluación del plan.

La comprensión del problema es considerada esencial en todos los métodos, tanto por su papel en la motivación como para la comprensión del enunciado del problema. Se comprende el problema cuando se es capaz de reproducirlo con las propias palabras y de analizar sus elementos esenciales, lo que se puede favorecer a través de impulsos en forma de preguntas que movilicen el pensamiento: ¿de qué trata el problema?, ¿qué datos se dan?, ¿qué se busca?, ¿seré capaz de resolverlo?, ¿son suficientes los conocimientos de que dispongo para buscar la vía de solución?, ¿ son suficientes los datos?

En esta fase es importante la identificación de conceptos y definiciones involucrados o relacionados con la información que brinda el problema, lo que es propio de la dimensión lógica. Es fructífero que el estudiante se entrene en la reflexión y el autocuestionamiento, lo que propicia su autocontrol y la capacidad para tomar determinadas decisiones que pueden determinar el curso del proceso de resolución, elementos propios de la dimensión metacognitiva.

Una segunda fase es la elaboración de un plan, comienza con la precisión del problema, se analizan los medios y se busca una idea de solución. Encontrar la idea o vía de 
solución es un proceso de deducciones, inducciones, análisis, y síntesis, resultado de la actividad mental desarrollada en la fase anterior.

Para esto, el docente puede brindar impulsos, en forma de sugerencias, que cuando son asumidas como hábitos facilitan la exploración de posibles vías de solución. Entre otros el docente puede sugerir: analiza todas las posibles deducciones que se pueden inferir de los datos del problema, ¿recuerdas otro problema similar o parecido que hayas resuelto?, construye tablas, esquemas o gráficos, ¿puedes formular alguna hipótesis sobre la solución del problema?, ¿de qué fórmula, expresión o modelo me puedo servir para hallar la solución?

En esta fase se puede organizar la información en tablas, esquemas u otros organizadores gráficos que faciliten el descubrimiento de relaciones que no son evidentes directamente, se exploran estrategias, se formulan hipótesis, se realizan acciones para obtener ideas sobre la vía de solución.

En la ejecución del plan concebido se concreta la solución del problema, en un proceso donde se articulan las deducciones y proposiciones pensadas de forma lógica y coherente, se validan las hipótesis formuladas anteriormente, entre otras acciones dirigidas a estructurar la vía de solución y satisfacer la exigencia del problema. Aquí, juega su papel la formación lingüística, las habilidades del estudiante para comunicar sus ideas a los demás, desarrollar la idea de solución de forma coherente, lógica y precisa.

Para lograr estos propósitos, el docente puede brindar impulsos como: demuestra si son válidas las hipótesis formuladas, realiza los cálculos necesarios, ¿qué ocurre si...? , construye tablas con la información del problema, representa la información en gráficos, entre otros.

La evaluación de la solución y de la vía es la valoración del plan desarrollado, en esta se predomina la metacognición, lo que no significa la ausencia del razonamiento lógico deductivo y la heurística.

En esta fase se garantiza la validez de la vía de solución desarrollada, se comprueba la vía de solución, si existen otras vías de solución alternativas, se señalan casos especiales, y la posibilidad de transferirla a otros problemas. Es la oportunidad para que el profesor estimule la reflexión y el pensamiento crítico con impulsos como: ¿es lógico el resultado?, ¿por qué?, ¿es posible comprobar la solución?, ¿cómo hacerlo?, ¿es posible resolver el problema por una vía más corta?, ¿qué otro resultado se puede obtener por esta vía?, ¿cómo llegué a la vía de solución? 
En realidad, a través de todo el proceso de resolución está presente la actividad valorativa y el control, desde que se comienza a leer reflexivamente el problema se van formando criterios acerca del mismo, sus exigencias y condiciones para la solución, estos criterios luego influirán en las decisiones que se toman en el proceso de búsqueda de la vía de solución: si es viable continuar con la idea de solución que se desarrolla o es necesario reorientar la misma ante obstáculos que no se tuvieron en cuenta inicialmente.

El desarrollo de la capacidad para la resolución de problemas matemáticos se potencia en la medida en que se combina con el estímulo de la capacidad para pensar en la resolución de problemas, es necesario que el estudiante desarrolle un modo de pensar que articule las dimensiones del pensamiento matemático, y, para ello, la instrucción heurística se puede convertir en una excelente estrategia de enseñanza.

A pesar de que los métodos de resolución de problemas se concretan en procedimientos estructurados en un orden, no deben ser utilizados como patrones rígidos, porque la búsqueda de la vía de solución puede necesitar de avances y retrocesos, ahí la razón de concederles más flexibilidad, teniendo en cuenta en cada fase el razonamiento lógicodeductivo, la heurística y la metacognición.

El pensamiento matemático es una cualidad necesaria en la mayoría de las esferas de la vida, aun más en un mundo que se informatiza constantemente, pero más necesaria aún en la formación del profesional de las ciencias técnicas. Por esta razón, vamos a ejemplificar las ideas propuestas en un problema para el cálculo de la fracción molar en la ingeniería química, aplicando métodos numéricos en una ecuación diferencial ordinaria.

\section{Ejemplo:}

Para diseñar una torre de destilación, se simula un destilador discontinuo a escala de laboratorio al que se alimenta 100 moles de una mezcla de $n$-heptano y otros compuestos $\left(\mathrm{S}_{0}\right)$. La fracción del n-heptano (compuesto que se destila), en la alimentación es igual a 0,7500 $\left(X_{h}\right)$. Al finalizar la destilación en 1 hora, quedan 10 moles de mezcla en el equipo $\left(S_{f}\right)$.

Aplicando un balance de masa en el destilador se obtuvo el siguiente balance de masa:

$$
\frac{\partial X_{h}}{\partial t}=1,16\left[\frac{S_{f}-S_{n}}{S_{f}(1-t)+S_{0}} \frac{X_{h}\left(1-X_{h}\right)}{1+1.16 X_{h}}\right] \text {. }
$$

Se necesita saber (con un error absoluto igual o menor que 0.1000 ), empleando un método numérico de al menos primer orden, si la fracción molar del n-heptano es inferior a 0.5000 al finalizar el tiempo de destilación, calculando como mínimo un punto intermedio en el intervalo de integración. 
Si la fracción molar del n-heptano es superior al 0.5000 , hay que modificar las condiciones de destilación.

Para estimular el desarrollo del pensamiento matemático en la resolución del problema es necesario que, en cada fase del proceso, se activen cada una de sus dimensiones a través de impulsos del docente en forma de reglas o preguntas. A continuación, se ofrecen algunas propuestas a modo de ejemplo con que el docente puede guiar la actividad mental de sus estudiantes utilizando el modelo de resolución de problemas:

$\checkmark$ Comprensión del problema

Procedimientos lógicos

- Lee detalladamente el Problema.

- Identifica palabras claves que expresen relaciones en el problema.

- Identifica las variables que intervienen en el problema.

- Expresa con tus palabras la idea fundamental del problema.

- ¿Cuáles de los datos son necesarios para encontrar la solución?, ¿Son suficientes estos datos?

- ¿En qué unidades se debe expresar el resultado, o es adimensional?

Procedimientos Heurísticos

- ¿Según la información de que se dispone, de qué tipo de problema se trata?

- ¿Qué se necesita encontrar para responder a la pregunta?

- ¿Recuerdo haber resuelto otro problema en las mismas condiciones?

Procedimientos Metacognitivos

- ¿Con mis conocimientos podré resolver el problema?

- ¿Cómo aparece representada la información sobre las variables que intervienen en el problema?

- ¿Cómo debo representar la información que se pide en el problema?

- ¿De qué conocimientos dispongo para encontrar la solución?

- ¿Puedo resolverlo con los conocimientos que tengo sobre el tema o me falta algo por conocer para resolverlo?

Elaboración de un plan

Procedimientos lógicos

- Si se exige como mínimo un punto intermedio, ¿con qué valores del tiempo se deben calcular las fracciones molares?,

- si se toma un paso $h=0.5$ entonces lo valores de $t$ son $t_{0}=0, t_{1}=0.5$, $t_{2}=1$, 


\section{Procedimientos Heurísticos}

- ¿Qué método es el más adecuado para buscar la solución?

- ¿Cuáles son las ecuaciones de trabajo con que se puede resolver el problema?:

$X_{h_{i+1}}=X_{h_{i}}+h\left[1.16 \frac{S_{f}-S_{0}}{S_{f}(1-t)+S_{0} t} \frac{X_{h_{i}}\left(1-X_{h_{i}}\right)}{1+1.16 X_{h_{i}}}\right]$;

$t=t+h$

- ¿Cuántas repeticiones serán necesarias para resolver el problema?

- ¿Cuáles son las condiciones iniciales para encontrar la solución del problema?

Procedimientos Metacognitivos

- ¿Qué puede ocurrir si se disminuyera el paso?, ¿y si aumentara?

$\checkmark$ Ejecución del plan

Procedimientos lógicos

- ¿Por qué utilizar ese método y esas ecuaciones?

- Piensa en el procedimiento resolver el problema con esas ecuaciones de trabajo, caso necesario descríbelos.

Procedimientos Heurísticos

- Sustituye los datos y calcula con las ecuaciones de trabajo.

- Calcula las iteraciones que se exigen.

- Verifica si se satisface el error permisible.

\section{Procedimientos Metacognitivos}

- ¿Esa solución responde a lo que se pide en el problema?

- ¿No hay contradicción con lo que pensaste inicialmente?

Evaluación del plan

Procedimientos lógicos

- ¿Es correcto el resultado?

- ¿No hay contradicciones con las condiciones iniciales?

Procedimientos Heurísticos

- Compara la vía de solución con las de otros problemas resueltos anteriormente,

Procedimientos Metacognitivos

- ¿Es correcta la vía empleada para resolver el problema?

- Piensa si se puede resolver por una vía mejor.

- Revisa todos los pasos para llegar a la vía de solución. 
- ¿Se puede obtener una solución más precisa?

El proceso de enseñanza aprendizaje de la Matemática debe potenciar que el estudiante aprenda a aprender, proporcionando estrategias para relacionar lo aprendido con los nuevos contenidos, realizar nuevos aprendizajes a través de su propia experiencia y desarrollar el pensamiento matemático.

De modo general, el papel de la educación es el de crear desarrollo, pero una educación se dice que es desarrolladora si promueve y potencia aprendizajes desarrolladores. El verdadero significado de esta categoría, y su trascendencia exige un proceso de continuo mejoramiento de la educación, pero es necesario esclarecer cuándo la enseñanza es desarrolladora.

Autores como Silvestre (2001), Rivero y Cuenca (2005), Castellanos et al. (2001), exponen que el aprendizaje desarrollador propicia que el estudiante participe activa, consciente y reflexivamente bajo la dirección del docente en la apropiación de conocimientos, habilidades, procedimientos y estrategias para actuar en interacción y comunicación con los demás, adquiriendo valores, sentimientos y normas de conducta.

Supone, además, la independencia cognoscitiva y la autorregulación de modo que los estudiantes aprendan a aprender. Debe garantizar, además, la apropiación activa y creadora de la cultura, propiciando un automejoramiento constante, la autonomía y autodeterminación, en el marco de procesos de socialización, compromiso y responsabilidad con la sociedad.

Los programas heurísticos, como sistema de medios y procedimientos para la búsqueda de solución a los problemas, constituyen una importante herramienta en manos del docente si son utilizados, además, como recurso para estimular el desarrollo del pensamiento matemático y favorecer la enseñanza y el aprendizaje desarrolladores.

\section{Metodología}

La mayor preocupación de los docentes se ha centrado en la meta de que el estudiante desarrolle o mejore la capacidad para resolver problemas, sin embargo con el desarrollo del pensamiento matemático, consecuentemente, se desarrollará esta capacidad, lo que es asumido como hipótesis de trabajo.

El objetivo de la puesta en práctica es la validación de la factibilidad del programa heurístico para estimular el desarrollo del pensamiento matemático. Este trabajo se desarrolla en el marco del proyecto Aprendizaje basado en problemas en el proceso de enseñanza 
aprendizaje de la ingeniería, con la finalidad de estimular el desarrollo del pensamiento a través de la resolución de problemas en la formación de ingenieros.

Para probar la hipótesis de trabajo se utiliza un pre-experimento (SAMPIER, 2003), lo cual consiste en aplicar una evaluación previa antes de desarrollar la experiencia, se miden los resultados teniendo en cuenta las dimensiones del pensamiento matemático ya declaradas, los que se tabulan y procesan.

A continuación, se desarrolla la experiencia a través de dieciséis clases prácticas de resolución de problemas, en dos temas del programa. Luego de la experiencia, se aplica una segunda evaluación. Las mediciones se realizan conforme a los indicadores de cada una de las dimensiones declarados anteriormente.

El estímulo del desarrollo del pensamiento matemático se concreta a través del programa heurístico en las clases prácticas de resolución de problemas, como ha sido ejemplificado anteriormente. El docente debe planificar los impulsos que brinda a los estudiantes, tomando como marco el programa heurístico, de modo que a través del proceso de resolución se estimulen las dimensiones esenciales del pensamiento matemático: el razonamiento lógico-deductivo, la heurística la metacognición.

La experimentación se desarrolla en la asignatura Métodos Numéricos en el segundo año de la facultad de ingeniería química de la Universidad Tecnológica de La Habana. Para demostrar la hipótesis asumida, se seleccionó como muestra 126 estudiantes, con estos se utilizó el programa heurístico, estimulando a través de este las dimensiones del pensamiento matemático.

El análisis de los datos va dirigido a verificar si con el estímulo de las dimensiones del pensamiento matemático enmarcadas en un modelo de resolución de problemas es posible estimular el desarrollo de este en los estudiantes.

Se utiliza la prueba paramétrica de Wilcoxon ${ }^{1}$ con los resultados de las mediciones en la muestra tomada, lo que permite establecer comparaciones entre el desarrollo del pensamiento matemático en los estudiantes antes y después de haber sido estimulados con los impulsos propuestos para activar las dimensiones del pensamiento matemático mediante el modelo de resolución de problemas asumido.

Para el procesamiento de los datos y la prueba se emplea el programa MINITAB 16, un programa informático que permite ejecutar funciones estadísticas tanto básicas como

\footnotetext{
${ }^{1}$ El test de Wilcoxon es una prueba no paramétrica para comparar las medianas de una muestra en un antes y un después y determinar si existen diferencias entre ellas. Se utiliza como hipótesis nula que la mediana de la muestra $(\eta)$ es igual a un valor hipotético $\left(\mathrm{H}_{0}: \eta=\eta_{0}\right)$, frente a la hipótesis alternativa de que la mediana de la muestra es menor que el valor hipotético.
} 
avanzadas. Con su ayuda se da tratamiento estadístico a los datos de las evaluaciones aplicadas, asumiendo un nivel de significatividad del 95\%, se parte de la hipótesis nula: mediana $\eta=65$ contra la alternativa $\eta>65$.

Los resultados arrojan una probabilidad $p=0.001$ menor que 0.95 por lo que se rechaza la hipótesis nula y se acepta la alternativa, de lo que se infiere que, luego de la utilización del modelo para estimular el desarrollo del pensamiento matemático en las clases de resolución de problemas, se observa un aumento cualitativo y cuantitativo del desarrollo deseado en las dimensiones referidas a partir de los indicadores asumidos.

Es importante destacar, como complemento a las conclusiones inferidas de los resultados numéricos, que a través de la experimentación se observa como, de forma gradual, los estudiantes van incorporando como hábitos las acciones estimuladas por el docente y van haciendo suyas formas de pensar propias del pensamiento matemático.

\section{Consideraciones finales}

El desarrollo acelerado de la ciencia y la tecnología demanda de la educación la formación y desarrollo en los estudiantes del pensamiento matemático. Una importante vía puede ser el empleo intencional de los métodos de resolución de problemas matemáticos, aprovechando los recursos de la heurística de modo que el docente estimule desde el plano externo las dimensiones propias del pensamiento matemático a través de impulsos heurísticos y el estudiante las internalice de forma gradual.

En la investigación se constató, en la práctica educativa, la aplicación de métodos de resolución de problemas para estimular el desarrollo del pensamiento matemático que, de hecho, implica el desarrollo de la capacidad para resolver problemas.

Los resultados se corresponden con la tendencia a prestar más atención al desarrollo de la capacidad para pensar a través de la resolución de problemas. Desde esta perspectiva, el estudiante no debe ser concebido como un sujeto que sigue un conjunto de pasos para resolver problemas, sino como el sujeto activo que moviliza y desarrolla su pensamiento matemático en la búsqueda de vías de solución a los problemas.

Al docente corresponde el papel de implementar acciones, impulsos heurísticos y procedimientos en forma de indicaciones, sugerencias o preguntas que movilicen la actividad mental de los alumnos en especial el pensamiento matemático.

\section{Referencias}


BALLESTER, S., H. et al. Metodología de la enseñanza de la Matemática. Tomo I. 1.ed. La Habana: Editorial Pueblo y Educación, 2001. 459 p.

CAPOTE, M. Una estructuración didáctica para la etapa de orientación en la Solución de problemas aritméticos con texto en el primer ciclo de la escuela primaria. 2003. 120f. Tesis (Doctorado en Ciencias Pedagógicas) - Universidad "Hermanos Saíz Montes de Oca", Pinar del Rio, 2003.

CASTELLANOS, D. et al. Hacia una concepción del aprendizaje desarrollador. 1. ed. La Habana: Instituto Superior Pedagógico "Enrique José Varona”, 2001. 97p.

FERNÁNDEZ, J. A. Estrategias de enseñanza y aprendizaje: formación del profesorado y Técnicas creativas para la resolución de problemas matemáticos. 1. ed. Barcelona: CISSPRAXIS, 2003. 195 p.

GUILERA, L. Vías de acceso conceptual en la resolución de problemas. Importancia de los estímulos sensoriales. 2002, 120f. Tesis (Doctorado en Ciencias Psicológicas) - Universidad Autónoma de Barcelona, España, 2002.

KOLIAGUIN, Y. M. Metodología de la enseñanza de la Matemática en la escuela media. 1. ed. Moscú: Editorial Instrucción, 1975. 128p.

KRULIK, S.; RUDNICK, J. A. Problem Solving: A Handbook for Elementary School Teachers. 2. ed. Massachusetts: Allyn and Bacon, Inc, 1988. 248p.

MINISTERIO DE EDUCACIÓN. En: IV Seminario Nacional a Dirigentes Metodólogos, Inspectores y Personal de los Órganos Administrativos de las Direcciones Provinciales y Municipales de Educación (Documentos Normativos y Metodológicos) IV PARTE, 4., 1980, La Habana, Cuba. Anales. La Habana: Editorial del Ministerio de Educación, 1980. 50 p.

MÜLLER, H. El trabajo heurístico y la ejercitación en la enseñanza de la Matemática en la enseñanza general, politécnica y laboral. 1978. 80f. Disertación (Metodología de la Enseñanza de la Matemática) - Instituto Superior Pedagógico "Frank País García”, Santiago de Cuba, 1978.

NATIONAL COUNCIL OF TEACHERS OF MATHEMATICS. Principles and Standards for School Mathematics. 1. ed. Virginia, 2010. 9 p.

. Commission on Standards for School Mathematics. Curriculum and Evaluation Standards Report. 2004. 12 p.

POLYA, G. How solve it. A New Aspect of Mathematical Method. 2. ed. New Jersey: Princeton University Press, 1973. 272 p.

ONUCHIC, L. R.; ALLEVATO, N. S. G. Novas reflexões sobre o ensino-aprendizagem de matemática através da resolução de problemas. En: BICUDO, M. A. V.; BORBA, M. C. (Org.). Educação Matemática: pesquisa em movimento. Sao Paulo: Cortez, 2004. 25 p.

RODRÍGUEZ, J. B. Una Propuesta Metodológica para la utilización de las tecnologías de la información y las comunicaciones en el proceso de enseñanza-aprendizaje de las funciones matemáticas. 2003. 120f. Tesis (Doctorado en Ciencias Pedagógicas) - Instituto Superior Pedagógico "Enrique José Varona”, La Habana, 2003.

RON, J. Una Estrategia Didáctica para el proceso de enseñanza-aprendizaje de la Resolución de Problemas en las clases de Matemática en la educación Secundaria Básica. 2007. 120f. Tesis (Doctorado en Ciencias Pedagógicas) - Instituto Superior Pedagógico "Enrique José Varona”, La Habana, 2007. 
RIVERO, M.; CUENCA, M. Educación en la diversidad para una enseñanza desarrolladora, Curso 31. Pedagogía 2005. 1. ed. La Habana: Editorial Educación Cubana, 2005. 48 p.

SAMPIER, R. Metodología de la Investigación. Tomo 1. 2. ed. La Habana: Editorial Félix Varela. 2003. $469 \mathrm{p}$.

SANTOS, M. S. A metodologia de resolução de problemas como atividade de investigação: um instrumento de mudança didática. 1993. 253f. Tese (Doutorado em Educaçao) - Faculdade de Educação, Universidade de São Paulo, São Paulo, 1993.

SAUSEN, S., GUÉRIOS, E. Licenciatura em matemática: resolução de problemas na disciplina de metodologia do ensino com utilização das TICs. En: ENCONTRO NACIONAL DE EDUCAÇÃO MATEMÁTICA, 10., 2010, Salvador. Anais do X Encontro Nacional de Educação Matemática. Salvador: ENEM, 2010.

SCHOENFELD, A. H. Mathematical Problem Solving. 1. ed. London: Academic Press. Inc., $1985.424 \mathrm{p}$.

SCHOENFELD, A. H. Learning to think mathematically: problem solving, metacognition, and sensemaking in mathematics. En: GROUWS, D. (Ed.). The Handbook for Research on Mathematics Teaching and Learning. New York: Mac Millan, 1992. 20 p.

SILVESTRE, M. Aprendizaje, educación y desarrollo. 1. ed. La Habana: Editorial Pueblo y Educación, 2001. 117 p.

SUÁREZ, C. La identificación de problemas matemáticos en la educación primaria. 2003, 120f. Tesis (Doctorado en Ciencias Pedagógicas) - Instituto Superior Pedagógico "Enrique José Varona". La Habana, Cuba, 2003.

VILA-CORTS, A. Resolució de problemas de Matemátiques: identificació, origen i formació del sisteme des creences en l'alumnat. Alguns efectes sobre l'abordatge dels problemas. 2001, 774f. Tesis (Doctorado en Ciencias Pedagógicas) - Universidad Autónoma de Barcelona, Barcelona, 2001.

WANG, K. Implications from Polya and Krutetskii. En: INTERNATIONAL CONGRESS ON MATHEMATICAL EDUCATION. 12., Seoul, 2012. Anais... Korea: COEX, 2012. 12 p.

ZUFFI, E. M.; ONUCHIC, L. R. O Ensino-Aprendizagem de Matemática a través da Resolução de Problemas e os Processos Cognitivos Superiores. Unión- Revista Iberoamericana de Educación Matemática, n. 11, p. 79-97, set. 2007. Disponible en: <www.fisem.org/www/union/revistas/2007/ .../Union_011_009.pdf >. Acceso en: 10 feb. 2012.

Submetido em 29 de Junho de 2016. Aprovado em 17 de Outubro de 2016. 